\title{
Subject Index Vol. 17, 1996
}

Acute-phase plasma proteins 281

Adenocarcinoma 345

Alkaline phosphatase 320

Amplification 262

Analytical electron microscopy 234

Antibodies, monoclonal 196

Antibody affinity 196

- $\quad$ specificity 196,325

di-Antichymotrypsin 281

Antiestrogen 48

Apoptosis 354

Ascites 1

B16F10 melanoma 155 Basement membrane 48 be $1-2$ oncoprotein 341

- proto-oncogene 65

Benign prostatic hyper

trophy 20

Breast cancer 5, 110

- cell lines 290

tumors 299

CA15-3 110 CA125 1,90

- $\quad$ antigen 196,325

Carcinoembryonic antigen

58 Catecholamine mass

screening, urinary 65 Cathepsin-D 290 Cell adhesion 117

proliferation 155

viability 354 Choriocarcinoma 13 Chromosome lp deletions

65 Clinical performance 110

stages 226 Colon cancer 75

carcinoma 58 Combined histologic types

341 Computer simulation 133 Cryopreparation 234 Cryosections 234 Cytogenetics 27

Cytotoxicity 354

D14 monoclonal antibody

58 Decidua 13 Dibutyryl cyclic AMP 34

Differentiation 5, 226 Diffuse malignant mesothe-

lioma 1 Digital image analysis 306 Double minutes 65 Drug metabolism, hepatic 102

- $\quad$ targeting 234

Ductal carcinoma, infil

trating 5 
Dye exclusion assays 354

Electron energy loss spec-

troscopy 234 Endometrial cancer 48, $226 \gamma$-Enolase 271 Epidermal growth factor

168 Epitope mapping 196 Estrogen 48 Extracellular cell matrix 251

- $\quad$ matrix 117,332

$\alpha$-Fetoprotein 251,299 Fibroblast growth factors

226 Field effect 58 5-Fluorouracil 306

Gastric cancer 281 Gene 299

$\gamma$-Glutamyltransferase 369 Glycosylation 176 Granulated metrial gland

cells 13 Growth factor 290

Ha-ras proto-oncogene 65 Head and neck tumors 81 Heparin 345 Homeobox genes 34

Homogeneously staining

regions 65 Hormone dependence 290 Human 251,299

- neuroblastoma 34

Hybridization 299

Immune response 133 Immunohistochemistry 5,

58,325,341 Immunosuppressive acidic

protein 281 Immunotherapy 27 Integrins 117

Interleukin-2 expression 155

- receptor 155

Invasion 125, 133, 168,332

Invasiveness 48

Isoenzymes 271

Keratinocyte 97

Laminin 125, 332 Lung neoplasms 176 Lymph node metastasis 281 Lymphoma 362

MAbTJAC4 5 Malignant melanoma 271 Mast cells 345 Mathematical modelling

133 Meconium 320 Mediastinal tumors 362 Metastasis 125,332 Monoclonal antibodies 5, 369 mRNA 226 Mucin 176 Murine sarcoma 125 Myoblasts 251 Myometrial invasion 226

Myotubes 251

Neovascularization 226 Neuroblastoma 65, 262 Neuron-specific enolase

271,362 Neuronal differentiation $34 \mathrm{~N}$-myc 262

amplification 65

oncoprotein 65

Oral cancer 168 Osteosarcoma 332 Ovarian neoplasms 90

$\mathrm{pH}$, intracellular 133 -, microenvironmental

133 Ploidy 75 Polymerase chain reaction

262 Prognosis 281 Prognostic factors 75

- value 81

Prostate cancer 20

Prostate-specific antigen

density 20 pS2 290

Receptors 251

Recurrence 75

Reference range 110

Regeneration 220

Renal cell carcinoma 27, 369 


\section{Retinoicacid 34}

Reverse transcriptase polymerase chain reaction, competitive 30

S stage arrest 220 SCC antigen 81 Selenium 102 Signal transduction 117,

133 Small-cell lung cancer 341 ,

362

- $\quad$ - - cell lines 271

Squamous cell carcinoma(s)

81,168

- - antigen 97

epithelium, normal 97 Sugar chain 320 Superfibronectin 117 Survival 75

Teratoma 362 Tetrazolium salts 354 Thymidylate synthase 306 Thymoma 362 TRK protooncogene 65 Trophoblast 13 Trypanblue 354 Tumor cell growth 345

growth 133

invasion 281

marker(s) 1,20,81,110, 196

necrosis factor receptor 90

- $\quad$ - factor- $\alpha 97$

Tumor-associated antigen

81,176 Tumoricidal activity 354 Type IV collagen 125

Urodeles 220

380 